\title{
Young-onset and late-onset Parkinson's disease exhibit a different profile of fluid biomarkers and clinical features
}

\author{
Tommaso Schirinzi ${ }^{\mathrm{a}, \mathrm{b}, *}$, Giulia Di Lazzaro ${ }^{\mathrm{a}}$, Giulia Maria Sancesario ${ }^{\mathrm{c}, \mathrm{d}}$, \\ Susanna Summa ${ }^{\mathrm{b}}$, Simona Petrucci ${ }^{\mathrm{e}, \mathrm{f}, \mathrm{g}}$, Vito Luigi Colona ${ }^{\mathrm{a}}$, Sergio Bernardini ${ }^{\mathrm{d}}$, \\ Mariangela Pierantozzi ${ }^{\mathrm{a}}$, Alessandro Stefani ${ }^{\mathrm{a}}$, Nicola Biagio Mercuri ${ }^{\mathrm{a}, \mathrm{c}}$, \\ Antonio Pisani $^{\mathrm{a}, \mathrm{c}}$ \\ ${ }^{a}$ Department of Systems Medicine, University of Roma Tor Vergata, Rome, Italy \\ ${ }^{\mathrm{b}}$ Department of Neurosciences, IRCCS Bambino Gesù Children's Hospital, Rome, Italy \\ ${ }^{\mathrm{C}}$ Department of Experimental Neurosciences, IRCCS Santa Lucia Foundation, Rome, Italy \\ ${ }^{\mathrm{d}}$ Department of Experimental Medicine and Surgery, University of Roma Tor Vergata, Rome, Italy \\ e Department of Clinical and Molecular Medicine, Sapienza University of Rome, Rome, Italy \\ ${ }^{\mathrm{f}}$ Department of Clinical and Molecular Medicine, S. Andrea University Hospital, Rome, Italy \\ ' IRCCS Casa Sollievo della Sofferenza, San Giovanni Rotondo, Italy
}

\section{A R T I C L E I N F O}

\section{Article history:}

Received 22 October 2019

Received in revised form 10 February 2020

Accepted 12 February 2020

Available online 18 February 2020

Keywords:

Young-onset Parkinson's disease

Early-onset Parkinson's disease

Late-onset Parkinson's disease

Aging

Fluid biomarkers

\begin{abstract}
A B S T R A C T
Young-onset Parkinson's disease (YOPD) is a relevant condition whose neurobiology is questioned if different from those of typical late-onset Parkinson's disease (LOPD). Here, we explored whether the clinical-biochemical profile of Parkinson's disease (PD) could be affected by the age-of-onset (AO), as a possible result of a distinct neurodegenerative process. A panel of fluid biomarkers (CSF lactate, 42amyloid- $\beta$ peptide, total and 181-phosphorylated tau; serum uric acid) and the standard scores for motor and nonmotor signs were assessed in 76 idiopathic PD patients (genetic cases excluded; YOPD, AO $\leq 50, \mathrm{n}=44$; LOPD, $\mathrm{AO}>50, \mathrm{n}=32$ ) and 75 sex/age-matched controls, adjusting the models for the main confounding factors. In PD, AO directly correlated to either CSF lactate and tau proteins or the nonmotor symptoms scale score. Specifically, a younger AO was associated with lower levels of biomarkers and minor burden of nonmotor symptoms. Our findings indicate that clinical-biochemical features of idiopathic PD may vary depending on the AO, accounting for different profiles in YOPD and LOPD whose recognition is fundamental for further pathophysiological implications and clinical applications.
\end{abstract}

(c) 2020 Elsevier Inc. All rights reserved.

\section{Introduction}

Idiopathic Parkinson's disease (PD) is a common disorder of elderly; however, much rarely it could occur in younger age $(\leq 50)$, as "young-onset PD" (YOPD) (Mehanna and Jankovic, 2019; Willis et al., 2013). At present, the worldwide incidence of YOPD is significantly increasing (Willis et al., 2013; Ylikotila et al., 2015), although it almost remains a neglected condition. Indeed, the ageof-onset (AO)-based classification of PD is still controversial, and univocal cutoffs to label YOPD are still lacking (Mehanna and Jankovic, 2019; Willis et al., 2013). Studies focusing on YOPD often include tout court idiopathic cases and monogenic parkinsonisms,

\footnotetext{
* Corresponding author at: Department of Systems Medicine, University of Rome Tor Vergata, Viale Oxford 1, 00139 Rome, Italy. Tel: +39 06 72596010; fax: +39 06 72596006 .

E-mail address: t.schirinzi@yahoo.com (T. Schirinzi).
}

which in turn have peculiar pathogenesis and phenotypes (Mehanna and Jankovic, 2019; Morgante et al., 2016). Nevertheless, the few available evidence describes a different clinicalpathological course between YOPD and typical-onset PD (>50 years, here "late-onset PD," LOPD), with a disproportionate burden of complications and quality of life (Liu et al., 2015; Mehanna et al., 2014; Mehanna and Jankovic, 2019; Pagano et al., 2016; Willis et al., 2013). YOPD thus deserves specific efforts to elucidate underlying pathogenic mechanisms, which is critical for the development of personalized treatments, urgently needed in such frail patients.

Neurodegenerative processes occurring at brain level may mirror in CSF and blood of patients; therefore, the assessment of fluid biomarkers represents a valuable tool to explore molecular mechanisms of the diseases in vivo (Alwardat et al., 2019; Bakshi et al., 2019; Liguori et al., 2015; Petrillo et al., 2020; Schirinzi 
et al., 2017, 2018a). Here, we thus hypothesized that the clinicalbiochemical profile of PD could by affected by the AO. We therefore analyzed a panel of fluid biomarkers (CSF A $\beta 42$, t-tau, p-tau, lactate, and serum UA) and standard clinical scores in a cohort of PD patients and age/sex-matched controls to evaluate the effect of $\mathrm{AO}$ and identify distinct patterns in YOPD and LOPD, which may inform on the underlying pathophysiology. Of relevance, to avoid confusion due to the mechanistic bases of the inherited forms of PD, we specifically focused on idiopathic cases.

\section{Methods}

The study was performed at Tor Vergata University Hospital (Rome, Italy) and involved 151 subjects, 76 PD patients and 75 controls (CTL), enrolled from an initial cohort of 168 subjects.

PD was diagnosed by using the 2015 Movement Disorder Society's criteria. Most frequent inherited cases of PD were ruled out (SNCA, LRRK2, PRKN, PINK1, DJ-1, and GBA), to avoid potential confounding factor (details in Supplementary File1).

CTL group included age/sex-matched subjects, with nonneurodegenerative conditions (psychogenic disorders [Hallett, 2016], headache, peripheral nervous system conditions) without signs of motor and cognitive impairment.

In agreement with previous studies (Schirinzi et al., 2018b), to improve diagnostic accuracy and avoid further potential biases, we excluded (1) subjects showing a CSF biomarkers profile suggestive of Alzheimer's disease $(A \beta 42 / p$-tau $<6.4)(n=2)$; (2) CSF samples with $>4$ cells; ( 3 ) subjects carrying conditions potentially affecting serum UA and CSF biomarkers concentrations (Schirinzi et al., 2017, 2018c) $(\mathrm{n}=12)$ (details in Supplementary File1); (4) subjects with overt cognitive decline (adjusted Mini-Mental State Examination, MMSE < 25) $(\mathrm{n}=3)$.

Depending on the AO (Willis et al., 2013), PD patients were grouped into YOPD ( $A O \leq 50, \mathrm{n}=44)$ and LOPD ( $\mathrm{AO}>50, \mathrm{n}=32$ ). Similarly, CTLs were divided into young CTL (YCTL, $\mathrm{n}=36$ ) and late CTL (LCTL, $\mathrm{n}=39$ ), to obtain 2 groups comparable with YOPD and LOPD (distribution of "control diagnoses" in YCTL and LCTL was similar).

Patients were enrolled close to the disease's onset. Clinicaldemographic data were recorded. At the time of sampling, a specialist systematically assessed PD patients under the effects of habitual antiparkinsonian medication (so called "ON" state) with the following: Unified Parkinson Disease Rating Scale part 2 and 3 (UPDRS II-III), Hoehn and Yahr scale (H\&Y), Non-Motor Symptoms Scale (NMSS), MMSE adjusted for age and educational level, Apathy Scale (by Starkstein et al., 1995); the type of antiparkinsonian drug was specified (levodopa, dopamine agonist, MAO inhibitor, or none) and the personal levodopa equivalent daily dose (LEDD) was calculated.

Blood/CSF sampling and biomarkers assay (CSF lactate, t-tau, ptau, $A \beta 42$, serum UA) were performed according to standard procedures, as previously described (Liguori et al., 2015; Schirinzi et al., 2018b,c) (Supplementary File1). The study was conducted in agreement with Helsinki declaration principles, with the approval of "Fondazione Policlinico Tor Vergata" ethical committee (0026092/2017). Informed consent was obtained from each participant.

\subsection{Statistical analysis}

The distribution of variables was evaluated with the ShapiroWilk test. Demographics were tested among the groups with parametric or nonparametric tests, as appropriate. Clinical and biochemical data non-normally distributed were Log10transformed.
Group differences in biomarkers were assessed by the one-way ANCOVA test, by using the single biomarker as dependent variable, the clinical group as fixed factor and sex as covariate. Group differences in clinical features were tested by a one-way ANCOVA model adjusted for sex, disease duration, and LEDD.

Prevalence of antiparkinsonian medications was expressed for each category as \% of the group and compared between YOPD and LOPD by chi-square test.

To identify a potential age-specific effect on biomarkers, a comparative regression analysis was conducted between PD and CTL, by using the biomarker as dependent variable, age of sample as independent variable, and sex as covariate. Then, to explore the effect of AO on both biomarkers levels and clinical parameters in PD patients, 2 different linear regression models were created, adjusting for main confounding factors: one using the single biomarker as dependent variable, AO as independent variable, sex and disease duration as covariates; the second using the clinical score as dependent variable, AO as independent variable, and sex, disease duration, and LEDD as covariates. Clinical-biochemical correlations were evaluated into clinical groups by the Spearman's test.

Statistical significance was set at $p<0.05$. Statistical analysis was performed in blind by using IBM-SPSS- 22 .

\section{Results}

\subsection{Comparative analysis of the whole PD and CTL populations}

Clinical-demographic and biochemical parameters of the whole PD population (YOPD+LOPD) were compared to the whole group of CTL (YCTL+LCTL) (Table 1). Age/sex distribution was homogeneous in both the groups. The one-way ANCOVA showed that levels of CSF lactate, serum UA, and CSF p-tau were similar (Supplementary File1); conversely, PD levels of CSF A $\beta 42$ and t-tau were significantly lower than CTL $(\mathrm{A} \beta 42: 2.87 \pm 0.17$ vs. $2.93 \pm 0.11 ; \mathrm{F}(1,82)=$ 5.17, $p=0.026$; partial $\eta^{2}=0.059$ ); (t-tau: $2.18 \pm 0.22$ vs. $2.29 \pm$ $0.20 ; \mathrm{F}(1,87)=5.54, p=0.021$; partial $\left.\eta^{2}=0.060\right)$.

\subsection{Effect of age on biomarkers on the whole PD and CTL populations}

Because biomarker levels may change depending on a pure age effect, this set of analysis was conducted to clarify the relationship between age and biomarkers comparatively in CTL and PD groups.

In CTL, only age directly correlated to serum UA levels $(\mathrm{T}=2.5$, $\left.p=0.017 ; \mathrm{F}(2,34)=7, p=0.003, \mathrm{R}^{2}=0.292\right)$. In PD, CSF lactate, $\mathrm{t}-$ tau, and p-tau directly correlated with age (respectively: CSF lactate $\mathrm{T}=2.189, p=0.033 ; \mathrm{F}(2,51)=4.75, p=0.013, \mathrm{R}^{2}=0.157 ; \mathrm{t}$-tau: $\mathrm{T}=$ 3.17, $p=0.003 ; \mathrm{F}(2,46)=9.12, p<0.001, \mathrm{R}^{2}=0.284 ; \mathrm{p}$-tau: $\mathrm{T}=$ 3.087, $p=0.003 ; \mathrm{F}(2,46)=5.62, p=0.007, \mathrm{R}^{2}=0.96$ ); no significance was instead obtained for CSF A 442 and serum UA (additional data in Supplementary File1).

\subsection{Effect of $A O$ on biochemical and clinical features of $P D$ patients}

Because the classification of PD patients in YOPD and LOPD is not univocally defined, this set of analysis explored how AO is related per se to biochemical and clinical parameters of the whole PD population, independently from main confounding factors.

\subsubsection{Biomarkers}

In $\mathrm{PD}$, we found a direct correlation between $\mathrm{AO}$ and either CSF lactate $\left(\mathrm{T}=2.23, p=0.03 ; \mathrm{F}(2,50)=4.77, p=0.013, \mathrm{R}^{2}=0.16\right)$, or CSF t-tau $\left(\mathrm{T}=2.61, p=0.012 ; \mathrm{F}(2,45)=7.24, p=0.002, \mathrm{R}^{2}=0.24\right)$ and $\mathrm{p}$-tau $\left(\mathrm{T}=2.71, p=0.009 ; \mathrm{F}(2,45)=4.42, p=0.018, \mathrm{R}^{2}=0.16\right)$, 
such as those patients with younger onset had lower levels of that biomarkers. Conversely, AO was not related to CSF A $\beta 42$ $(\mathrm{T}=-0.202, p=0.841)$ and serum $\mathrm{UA}(\mathrm{T}=0.241, p=0.811)$ (Fig. 1$)$.

\subsubsection{Clinical features}

AO was directly associated with NMSS $(\mathrm{T}=3.52, p=0.001$; $\left.\mathrm{F}(4,40)=3.44, p=0.017, \mathrm{R}^{2}=0.256\right)$, with a minor score in patients with young age-of-onset (Supplementary File1).

3.4. Comparative analysis among the subgroups (YOPD, LOPD, YCTL, $L C T L)$

In this set of analysis, the clinical-demographics and biochemical data of the 4 subgroups have been compared, to find distinctive patterns (Table 2). Age and gender distribution resulted similar between YOPD versus YCTL and LOPD versus LCTL.

\subsubsection{Biomarkers}

One-way ANCOVA test was run for every biomarker to compare YOPD versus LOPD; YOPD versus YCTL; LOPD versus LCTL.

CSF lactate of YOPD $(\log 10$ mean $\pm \mathrm{SD}=0.19 \pm 0.6)$ was similar to YCTL $(0.198 \pm 0.6)$, but significantly lower than LOPD $(0.238 \pm$ $0.07)\left(\mathrm{F}(1,51)=4.37, p=0.04\right.$; partial $\left.\eta^{2}=0.079\right)$. LOPD and LCTL $(0.27 \pm 0.07)$ did not differ.

CSF t-tau of YOPD $(2.1 \pm 0.19)$ was significantly lower than both $\operatorname{YCTL}(2.26 \pm 0.26)\left(\mathrm{F}(1,31)=3.88 p=0.05\right.$; partial $\left.\eta^{2}=0.111\right)$ and $\operatorname{LOPD}(2.21 \pm 0.24)\left(\mathrm{F}(1,46)=5.1, p=0.029\right.$; partial $\left.\eta^{2}=0.1\right)$. LOPD and $\operatorname{LCTL}(2.3 \pm 0.15)$ did not differ.

CSF p-tau of YOPD $(1.46 \pm 0.17)$ was similar to YCTL $(1.53 \pm 0.22)$ but significantly lower than that of LOPD $(1.58 \pm 0.19)(F(1,46)=$ 5.57, $p=0.023$; partial $\left.\eta^{2}=0.108\right)$. LOPD and LCTL $(1.6 \pm 0.12)$ did not differ. No group differences resulted for CSF A $\beta 42$ and serum UA levels (Supplementary File1).

\subsubsection{Clinical features}

MMSE was significantly higher in YOPD compared to LOPD (1.47 \pm 0.18 vs. $1.44 \pm 0.36 ; \mathrm{F}(1,30)=10.82, p=0.003$; partial $\left.\eta^{2}=0.265\right)$, while NMSS was lower $(1.34 \pm 0.39$ vs. $1.59 \pm 0.38, \mathrm{~F}(1,40)=3.85$, $p=0.05$; partial $\eta^{2}=0.088$ ) (Supplementary File1). A subanalysis on single NMSS items showed a higher score (more severe disorder) in item 7 (urinary function) for LOPD $(0.94 \pm 0.41$ vs. $0.49 \pm 0.30$; $\mathrm{F}(1,19)=4.43, p<0.05$; partial $\left.\eta^{2}=0.189\right)$. No differences resulted
PD

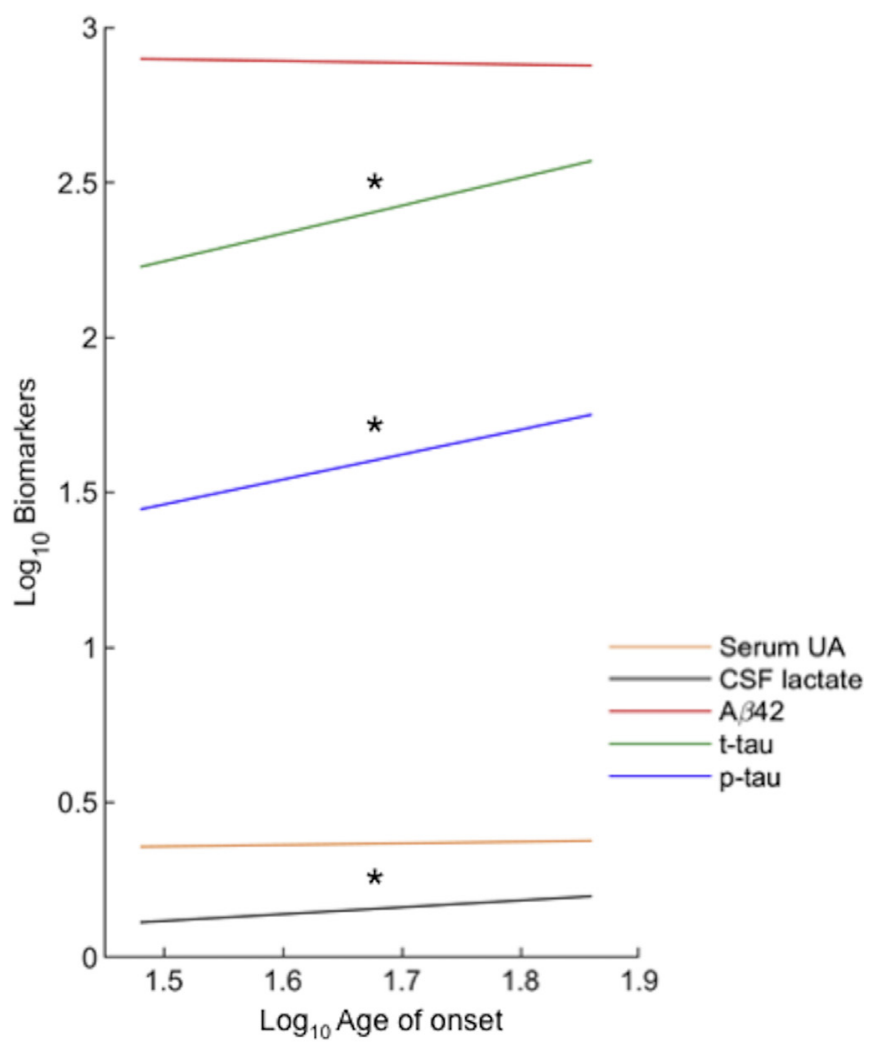

Fig. 1. The graph summarizes the regression lines resulted from the models testing the relationships between $\mathrm{AO}$ and biomarkers in PD population (both values are $\log 10$ transformed). Superimposed asterisks indicate statistical significance. Levels of CSF lactate and tau proteins increase in parallel of AO. Abbreviations: $\mathrm{AO}=$ age-of-onset; $\mathrm{PD}=$ Parkinson's disease.

in prevalence of antiparkinsonian medications between YOPD and LOPD (Supplementary File1).

\subsubsection{Correlation analysis}

In YOPD group, both CSF t-tau and p-tau levels were inversely correlated with NMSS $(\mathrm{R}=-0.6, p=0.038$ and $\mathrm{R}=0.6, p=0.042$, respectively).

Table 1

Clinical-demographic features of the study population (PD and CTL)

\begin{tabular}{|c|c|c|c|c|c|c|c|c|c|c|c|}
\hline \multirow[t]{2}{*}{ Variable } & \multirow[t]{2}{*}{ Significance } & \multicolumn{5}{|c|}{$\mathrm{PD}(\mathrm{n}=76, \mathrm{f}=44.7 \%)$} & \multicolumn{5}{|c|}{$\operatorname{CTL}(\mathrm{n}=75, \mathrm{f}=50.7 \%)$} \\
\hline & & Mean & SD & Median & Min & Max & Mean & SD & Median & Min & Max \\
\hline Age (y) & $p=0.154$ & 53.4 & 11.7 & 52.0 & 31.0 & 79.0 & 50.0 & 15.7 & 55.0 & 27.0 & 71.0 \\
\hline Sex & $p=0.604$ & & & & & & & & & & \\
\hline $\mathrm{AO}(\mathrm{y})$ & & 50.7 & 11.7 & 49.0 & 30.0 & 73.0 & - & - & - & - & - \\
\hline Duration (y) & & 2.5 & 2.1 & 2.0 & 0.0 & 9.0 & - & - & - & - & - \\
\hline CSF Lactate & $p=0.177$ & 1.7 & 0.3 & 1.7 & 1.1 & 2.7 & 1.7 & 0.3 & 1.7 & 1.1 & 2.4 \\
\hline CSF A $\beta 42(\mathrm{pg} / \mathrm{mL})$ & $p=0.026$ & 795.7 & 267.9 & 803.0 & 193.0 & 1555.0 & 902.0 & 241.7 & 919.0 & 458.0 & 1751.0 \\
\hline CSF t-tau (pg/mL) & $p=0.021$ & 171.7 & 91.8 & 150.0 & 39.0 & 484.0 & 213.2 & 89.6 & 213.0 & 49.0 & 542.0 \\
\hline CSF p-tau (pg/mL) & $p=0.424$ & 37.6 & 17.4 & 34.0 & 12.0 & 106.0 & 39.7 & 16.0 & 39.0 & 14.0 & 107.0 \\
\hline Serum UA $(\mathrm{mg} / \mathrm{dL})$ & $p=0.135$ & 4.1 & 1.2 & 4.3 & 1.4 & 6.5 & 4.5 & 1.2 & 4.4 & 2.3 & 7.4 \\
\hline MMSE & & 28.6 & 1.8 & 30.0 & 24.0 & 30.0 & - & - & - & - & - \\
\hline UPDRS II & & 6.8 & 3.7 & 7.0 & 1.0 & 13.0 & - & - & - & - & - \\
\hline UPDRS III & & 22.3 & 11.5 & 21.0 & 3.0 & 72.0 & - & - & - & - & - \\
\hline NMSS & & 35.1 & 28.8 & 27.5 & 0.0 & 140.0 & - & - & - & - & - \\
\hline Apathy scale & & 8.9 & 6.3 & 8.0 & 1.0 & 35.0 & - & - & - & - & - \\
\hline LEDD & & 143.1 & 216.2 & 0.0 & 0.0 & 920.0 & - & - & - & - & - \\
\hline H\&Y & & 1.7 & 0.51 & 2 & 1 & 2.5 & - & - & - & - & - \\
\hline
\end{tabular}

Significant $p$ value $(<0.05)$ in bold.

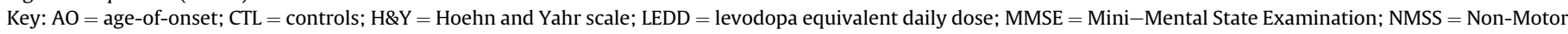
Symptoms Scale; PD = Parkinson's disease; UPDRS = Unified Parkinson Disease Rating Scale. 


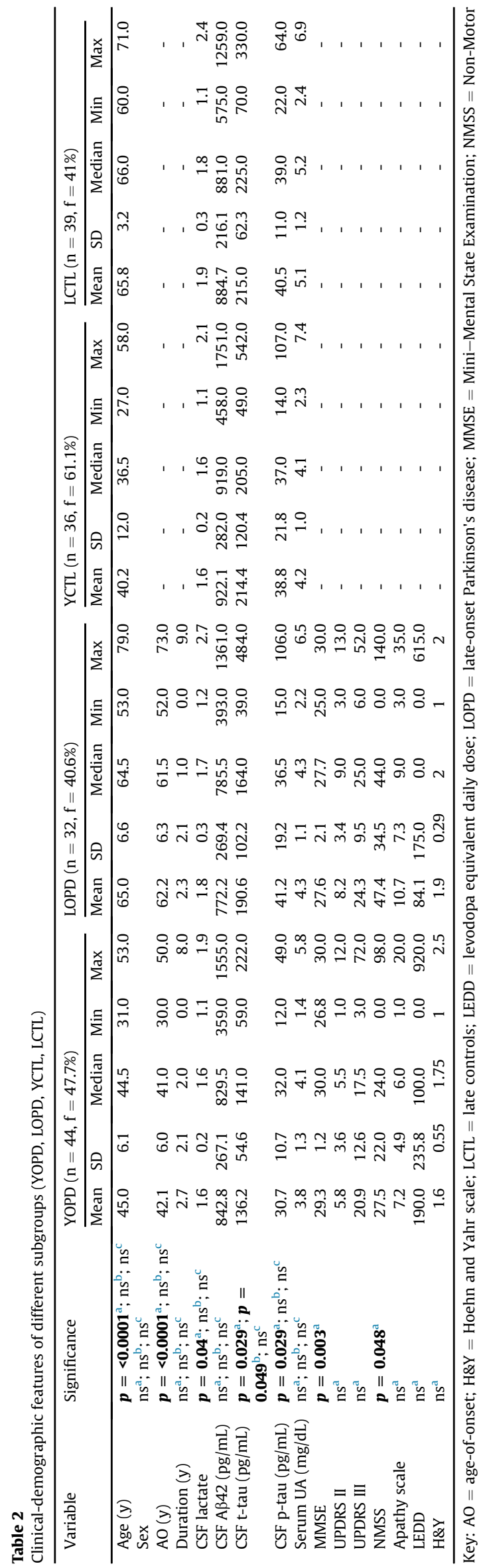

\section{Discussion}

This study explored whether clinical and biochemical features were affected by the AO in idiopathic PD. Of interest, we noticed that CSF levels of lactate and tau proteins (t-tau and p-tau), and the burden of nonmotor symptoms of PD patients increased with $\mathrm{AO}$, independently from the disease duration, accounting for different clinical-biochemical profiles in YOPD and LOPD. Here, patients have been assessed closely to their disease onset $(2.5 \pm 2.1$ years, mean \pm $\mathrm{SD})$, and thus, the associations between biomarkers and age overlapped those with AO. This indeed enabled a comparison with CTLs, which allowed us referring the clinical-biochemical findings of PD to early neuropathological events, rather than to aging or longlasting neurodegenerative processes.

CSF lactate is considered a readout of brain mitochondrial activity and redox balance (Liguori et al., 2015). Accordingly, its increase in parallel with AO indicates that bioenergetics' impairment, mitochondrial dysfunction, and oxidative stress, some of the major pathogenic milestones of PD (Schirinzi et al., 2016), gain progressive relevance in those patients with a later onset. Moreover, the agerelated increase of serum UA observed in CTL, but not in PD, seems to support this hypothesis. In fact, serum UA is a circulating antioxidant, whose levels have been inversely associated with the risk of PD and other neurodegenerative disorders (Bakshi et al., 2019; Schirinzi et al., 2017). In general, it is well established that the systemic oxidative stress aggravates with aging (Schirinzi et al., 2019b). Therefore, if healthy subjects maintain the neuroprotection mediated by UA, those subjects developing PD at later age lack this defense, being more vulnerable to oxidative stress.

Here we also observed that AO is associated to different CSF tau proteins level. In particular, we measured a reduction of t-tau and p-tau in PD compared to CTL; then, we found that levels were significantly lower in YOPD than in LOPD, with a direct association between protein content and AO. The reduction of CSF tau proteins has been already demonstrated in PD and other synucleinopathies (Kang et al., 2016; Mollenhauer et al., 2016; Pagano et al., 2016). The exact reason of such reduction is not understood yet, but it has been referred to molecular interactions between tau and alphasynuclein, occurring over the pathogenic cascade of the disease (Mollenhauer et al., 2016). Therefore, the difference in CSF tau proteins of YOPD and LOPD might reflect distinct molecular dynamics linked to neuronal damage, rather than a pure effect of age. Indeed, although it may be argued that the increase of CSF tau proteins in LOPD results from physiological aging (Li et al., 2017), we did not find significant age-related changes in the CTL group.

In synucleinopathies, CSF tau proteins are a direct index of global cerebral tau pathology (Gomperts et al., 2016; Irwin et al., 2018); in addition, their increase has been related to the disruption of the brain network connectivity and the progressive degeneration of Substantia Nigra (Abbasi et al., 2018; Zhang et al., 2016). Taking these findings together, we could hypothesize that the higher content of CSF tau proteins and the more severe burden of nonmotor symptoms, including the lower scores of MMSE (although within a range of normality), observed in LOPD patients, might underlie a greater brain accumulation of tau protein with more widespread neuropathology (Schirinzi et al., 2019a). Conversely, the lower relevance of these clinical-biochemical aspects in YOPD suggests a better preservation of neural systems, according with results from recent functional studies (Kübler et al., 2019).

The reduction of CSF A $\beta 42$ typically reflects cerebral amyloid plaques burden; although the levels may decrease with aging, they are considered a strong predictor of cognitive decline in PD patients (Alwardat et al., 2019; Mollenhauer et al., 2016; Schrag et al., 2017). As in previous reports (Goldman et al., 2018; Schrag et al., 2017), even in our cohort, the CSF A $\beta 42$ levels of PD patients were lower 
than CTL, probably for the enrollment of cognitively intact subjects, but at risk of future dementia. However, here, the AO was not related to $A \beta 42$ levels, and YOPD did not differ from LOPD. Unexpectedly, $A \beta 42$ neither correlated with MMSE, probably because of the enrollment criteria (exclusion of demented and old patients) and the relatively small sample of the population (Schrag et al., 2017).

This study has some limitations. The retrospective and crosssectional design prevented the longitudinal observation of clinical-biochemical parameters, while either the sample size, essentially due to the rarity of YOPD and to the scarcity of YCTLs, or the exclusion of demented patients might have influenced statistical analysis. Then, the absence of established definitions for YOPD and LOPD hindered classification of patients. However, we used strict inclusion criteria (of relevance the exclusion of inherited cases) and statistical models intended to limit such biases. Finally, we acknowledge the absence of other important biomarkers (alpha-synuclein and A $\beta 40$, especially) (Schirinzi et al., 2019a), which could have improved the whole study. Similarly, neuroimaging data could have been useful in integrating our clinical and biochemical data.

Despite such limitations, we show that AO can contribute to the clinical-biochemical profile of PD patients. Specifically, we found that a CSF marker of oxidative stress (lactate), the CSF levels of tau proteins, and the burden of nonmotor symptoms, which typically reflects the involvement of nondopaminergic systems, were more prominent in LOPD than in YOPD, independently from the disease duration, thus suggesting that different neurobiological substrates might underlie the 2 conditions. Overall, available data in this field are rather scarce yet. In a previous paper, Pagano et al. found that, in PPMI cohort (newly diagnosed, untreated PD patients), the CSF ttau levels increase in parallel with the AO of PD; as well, they observed that in those patients with older $\mathrm{AO}$, the motor and nonmotor symptoms were more severe and the dopaminergic dysfunction on 123I-ioflupane SPECT was greater (Pagano et al., 2016). Here, we included patients at the early stage of the disease, even under treatment, and idiopathic origin, because frequent genetic forms were excluded a priori. These peculiarities in inclusion criteria characterized our study, which indeed supports the idea that YOPD and LOPD may represent distinct subtypes of PD (Pagano et al., 2016), with different clinical and biochemical features, whose recognition is fundamental in the development of personalized treatments and, especially, in designing trials for neuroprotective drugs. Actually, biological aging may condition the evolution of PD, precipitating per se the molecular pathways involved in disease pathogenesis and thus worsening both the neuropathology and the clinical course (Collier et al., 2017).

At this regard, it seems urgent to improve research on this topic, even at preclinical level. In fact, major pathogenic determinants of YOPD remain still unknown. An exome sequencing study on YOPD patients revealed de novo variants in PTEN, VAPB, and ASNA1 genes, which accounts for cellular functions (e.g., trafficking and regulation of neural migration, number of apoptosis) (Kun-Rodrigues et al., 2015) different from the pathways typically involved in the pathogenesis of LOPD (e.g., mitochondrial or proteasomal dysfunction) (Schirinzi et al., 2016); however, the current evidence is almost poor.

\section{Conclusions}

Although preliminary, our results highlight significant clinicalbiochemical differences between YOPD and LOPD, which have to be considered in the development of personalized treatments or in the design of clinical trials for PD patients.

\section{CRediT authorship contribution statement}

Tommaso Schirinzi: Conceptualization, Methodology, Formal analysis, Writing - original draft. Giulia Di Lazzaro: Conceptualization, Writing - original draft. Giulia Maria Sancesario: Investigation, Resources, Methodology. Susanna Summa: Formal analysis, Software. Simona Petrucci: Investigation, Resources, Methodology. Vito Luigi Colona: Data curation, Software. Sergio Bernardini: Investigation, Resources, Methodology. Mariangela Pierantozzi: Conceptualization, Writing - review \& editing. Alessandro Stefani: Supervision, Writing - review \& editing. Nicola Biagio Mercuri: Supervision, Writing - review \& editing. Antonio Pisani: Conceptualization, Supervision, Writing - review \& editing.

\section{Acknowledgements}

All authors have reviewed the contents of the manuscript being submitted, approve of its contents, and validate the accuracy of the data.

This research did not receive any specific grant from funding agencies in the public, commercial, or not-for-profit sectors.

\section{Disclosure statement}

The authors declare no conflicts of interest.

\section{Appendix A. Supplementary data}

Supplementary data associated with this article can be found, in the online version, at https://doi.org/10.1016/j.neurobiolaging. 2020.02.012.

\section{References}

Abbasi, N., Mohajer, B., Abbasi, S., Hasanabadi, P., Abdolalizadeh, A., Rajimehr, R. 2018. Relationship between cerebrospinal fluid biomarkers and structural brain network properties in Parkinson's disease. Mov. Disord. 33, 431-439.

Alwardat, M., Schirinzi, T., Di Lazzaro, G., Sancesario, G.M., Franco, D., Imbriani, P., Sinibaldi Salimei, P., Bernardini, S., Mercuri, N.B., Pisani, A., 2019. Association between physical activity and dementia's risk factors in patients with Parkinson's disease. J. Neural Transm. 126, 319-325.

Bakshi, R., Macklin, E.A., Logan, R., Zorlu, M.M., Xia, N., Crotty, G.F., Zhang, E. Chen, X., Ascherio, A., Schwarzschild, M.A., 2019. Higher urate in LRRK2 mutation carriers resistant to Parkinson disease. Ann. Neurol. 85, 593-599.

Collier, T.J., Kanaan, N.M., Kordower, J.H., 2017. Aging and Parkinson's disease: different sides of the same coin? Mov. Disord. 32, 983-990.

Goldman, J.G., Andrews, H., Amara, A., Naito, A., Alcalay, R.N., Shaw, L.M., Taylor, P., Xie, T., Tuite, P., Henchcliffe, C., Hogarth, P., Frank, S., Saint-Hilaire, M.-H. Frasier, M., Arnedo, V., Reimer, A.N., Sutherland, M., Swanson-Fischer, C., Gwinn, K., , Fox Investigation of New Biomarker Discovery, U.J., Kang, U.J., 2018. Cerebrospinal fluid, plasma, and saliva in the BioFIND study: relationships among biomarkers and Parkinson's disease features. Mov. Disord. 33, 282-288.

Gomperts, S.N., Locascio, J.J., Makaretz, S.J., Schultz, A., Caso, C., Vasdev, N., Sperling, R. Growdon, J.H., Dickerson, B.C., Johnson, K., 2016. Tau positron emission tomographic imaging in the lewy body diseases. JAMA Neurol. 73, 1334.

Hallett, M., 2016. Functional (psychogenic) movement disorders-clinical presentations. Parkinsonism Relat. Disord. 22 (Suppl 1), S149-S152.

Irwin, D.J., Xie, S.X., Coughlin, D., Nevler, N., Akhtar, R.S., McMillan, C.T., Lee, E.B., Wolk, D.A., Weintraub, D., Chen-Plotkin, A., Duda, J.E., Spindler, M., Siderowf, A., Hurtig, H.I., Shaw, L.M., Grossman, M., Trojanowski, J.Q., 2018. CSF tau and $\beta-$ amyloid predict cerebral synucleinopathy in autopsied Lewy body disorders. Neurology 90, e1038-e1046.

Kang, J.-H., Mollenhauer, B., Coffey, C.S., Toledo, J.B., Weintraub, D., Galasko, D.R. Irwin, D.J., Van Deerlin, V., Chen-Plotkin, A.S., Caspell-Garcia, C., Waligórska, T., Taylor, P., Shah, N., Pan, S., Zero, P., Frasier, M., Marek, K., Kieburtz, K. Jennings, D., Tanner, C.M., Simuni, T., Singleton, A., Toga, A.W., Chowdhury, S., Trojanowski, J.Q., Shaw, L.M., Parkinson's Progression Marker Initiative, 2016. CSF biomarkers associated with disease heterogeneity in early Parkinson's disease: the Parkinson's progression markers initiative study. Acta Neuropathol. 131, 935-949.

Kübler, D., Schroll, H., Hamker, F.H., Joutsa, J., Buchert, R., Kühn, A.A., 2019. The effect of dopamine on response inhibition in Parkinson's disease relates to agedependent patterns of nigrostriatal degeneration. Parkinsonism Relat. Disord. 63, 185-190. 
Kun-Rodrigues, C., Ganos, C., Guerreiro, R., Schneider, S.A., Schulte, C., Lesage, S., Darwent, L., Holmans, P., Singleton, A., Bhatia, K., Bras, J., Bras, J., 2015. A systematic screening to identify de novo mutations causing sporadic earlyonset Parkinson's disease. Hum. Mol. Genet. 24, 6711-6720.

Li, G., Shofer, J.B., Petrie, E.C., Yu, C.-E., Wilkinson, C.W., Figlewicz, D.P., ShutesDavid, A., Zhang, J., Montine, T.J., Raskind, M.A., Quinn, J.F., Galasko, D.R., Peskind, E.R., 2017. Cerebrospinal fluid biomarkers for Alzheimer's and vascular disease vary by age, gender, and APOE genotype in cognitively normal adults. Alzheimers Res. Ther. 9, 48.

Liguori, C. Stefani, A Sancesario, G Sancesario G.M., Marciani, M.G., Pierantozzi, M. 2015. CSF lactate levels, $\tau$ proteins, cognitive decline: a dynamic relationship in Alzheimer's disease. J. Neurol. Neurosurg. Psychiatry 86, 655-659.

Liu, S.-Y., Wu, J.-J., Zhao, J., Huang, S.-F., Wang, Y.-X., Ge, J.-J., Wu, P., Zuo, C.-T., Ding, Z.-T., Wang, J., 2015. Onset-related subtypes of Parkinson's disease differ in the patterns of striatal dopaminergic dysfunction: a positron emission tomography study. Parkinsonism Relat. Disord. 21, 1448-1453.

Mehanna, R., Jankovic, J., 2019. Young-onset Parkinson's disease: its unique features and their impact on quality of life. Parkinsonism Relat. Disord. 65, 39-48.

Mehanna, R., Moore, S., Hou, J.G., Sarwar, A.I., Lai, E.C., 2014. Comparing clinical features of young onset, middle onset and late onset Parkinson's disease. Parkinsonism Relat. Disord. 20, 530-534.

Mollenhauer, B., Parnetti, L., Rektorova, I., Kramberger, M.G., Pikkarainen, M., Schulz-Schaeffer, W.J., Aarsland, D., Svenningsson, P., Farotti, L., Verbeek, M.M., Schlossmacher, M.G., 2016. Biological confounders for the values of cerebrospinal fluid proteins in Parkinson's disease and related disorders. J. Neurochem. 139 (Suppl 1), 290-317.

Morgante, F., Fasano, A., Ginevrino, M., Petrucci, S., Ricciardi, L., Bove, F., Criscuolo, C., Moccia, M., De Rosa, A., Sorbera, C., Bentivoglio, A.R., Barone, P., De Michele, G., Pellecchia, M.T., Valente, E.M., 2016. Impulsive-compulsive behaviors in parkin -associated Parkinson disease. Neurology 87, 1436-1441.

Pagano, G., Ferrara, N., Brooks, D.J., Pavese, N., 2016. Age at onset and Parkinson disease phenotype. Neurology 86, 1400-1407.

Petrillo, S., Schirinzi, T., Di Lazzaro, G., D’Amico, J., Colona, V.L., Bertini, E., Pierantozzi, M., Mari, L., Mercuri, N.B., Piemonte, F., Pisani, A., 2020. Systemic activation of Nrf2 pathway in Parkinson's disease. Mov. Disord. 35, 180-184.
Schirinzi, T., Di Lazzaro, G., Colona, V.L., Imbriani, P., Alwardat, M., Sancesario, G.M. Martorana, A., Pisani, A., 2017. Assessment of serum uric acid as risk factor for tauopathies. J. Neural Transm. 124, 1105-1108.

Schirinzi, T., Madeo, G., Martella, G., Maltese, M., Picconi, B., Calabresi, P., Pisani, A. 2016. Early synaptic dysfunction in Parkinson's disease: insights from animal models. Mov. Disord. 31, 802-813.

Schirinzi, T., Sancesario, G.M., Di Lazzaro, G., D’Elia, A., Imbriani, P., Scalise, S. Pisani, A., 2018a. Cerebrospinal fluid biomarkers profile of idiopathic normal pressure hydrocephalus. J. Neural Transm. 125, 673-679.

Schirinzi, T., Sancesario, G.M., Di Lazzaro, G., Scalise, S., Colona, V.L., Imbriani, P. Mercuri, N.B., Bernardini, S., Lang, A.E., Pisani, A., 2018b. Clinical value of CSF amyloid-beta-42 and tau proteins in Progressive Supranuclear Palsy. J. Neural Transm. 125, 1373-1379.

Schirinzi, T., Vasco, G., Zanni, G., Petrillo, S., Piemonte, F., Castelli, E., Bertini, E.S. 2018c. Serum uric acid in Friedreich Ataxia. Clin. Biochem. 54, 139-141.

Schirinzi, T., Sancesario, G.M., Di Lazzaro, G., Biticchi, B., Colona, V.L., Mercuri, N.B. Bernardini, S., Pisani, A., 2019a. CSF $\alpha$-synuclein inversely correlates with nonmotor symptoms in a cohort of PD patients. Parkinsonism Relat. Disord. 61, 203-206.

Schirinzi, T., Vasco, G., Aiello, C., Rizzo, C., Sancesario, A., Romano, A., Favetta, M. Petrarca, M., Paone, L., Castelli, E., Bertini, E.S., Cappa, M., 2019b. Natural history of a cohort of $A B C D 1$ variant female carriers. Eur. J. Neurol. 26, 326-332.

Schrag, A., Siddiqui, U.F., Anastasiou, Z., Weintraub, D., Schott, J.M., 2017. Clinical variables and biomarkers in prediction of cognitive impairment in patients with newly diagnosed Parkinson's disease: a cohort study. Lancet Neurol. 16, 66-75.

Willis, A.W., Schootman, M., Kung, N., Racette, B.A., 2013. Epidemiology and neuropsychiatric manifestations of young onset Parkinson's disease in the United States. Parkinsonism Relat. Disord. 19, 202-206.

Ylikotila, P., Tiirikka, T., Moilanen, J.S., Kääriäinen, H., Marttila, R., Majamaa, K., 2015 Epidemiology of early-onset Parkinson's disease in Finland. Parkinsonism Relat. Disord. 21, 938-942.

Zhang, Y., Wu, I.-W., Tosun, D., Foster, E., Schuff, N., Parkinson's Progression Markers Initiative, the P.P.M., 2016. Progression of regional microstructural degeneration in Parkinson's disease: a multicenter diffusion tensor imaging study. PLoS One 11, e0165540. 